\title{
CERTIFYING NON-REPRESENTABILITY OF MATROIDS OVER PRIME FIELDS
}

\author{
JIM GEELEN AND GEOFF WHITTLE
}

\begin{abstract}
It is proved that, for a prime number $p$, showing that an $n$-element matroid is not representable over $\operatorname{GF}(p)$ requires only $O\left(n^{2}\right)$ rank evaluations.
\end{abstract}

\section{INTRODUCTION}

We prove the following theorem.

Theorem 1.1. Let $p$ be a prime number. Then proving that an $n$ element matroid is not $\mathrm{GF}(p)$-representable requires at most $O\left(n^{2}\right)$ rank evaluations.

Theorem 1.1 is a corollary of a more technical result. This technical result — stated as Theorem 3.1 — gives sufficient conditions for a matroid to have a certificate of non-representability over a finite field using at most $O\left(n^{2}\right)$ rank evaluations. That these sufficient conditions are satisfied for prime fields follows from results in two other papers. The first is a theorem of Geelen and Whittle [6] that shows that matroids satisfying a certain strengthening of 3-connectivity have a bounded number of inequivalent $\mathrm{GF}(p)$-representations. The second is a theorem of Ben David and Geelen [2] that shows that excluded minors for $\mathrm{GF}(p)$-representability cannot have arbitrarily long nested sequences of separations of bounded order, in particular they cannot have long nested sequences of 3-separations. Theorem 3.1 is stated at a higher level of generality than we need for this paper. It is hoped that by doing this it may prove to be a useful tool in a future extension of Theorem 1.1 to the non-prime case.

Before proceeding to the technicalities we give some background to the problems addressed by this paper. In general it is not possible

1991 Mathematics Subject Classification. 05B35.

Key words and phrases. matroid, representable, prime field.

The first author was supported by a grant from the National Sciences and Engineering Research Council of Canada.

The second author was supported by a grant from the Marsden Fund of New Zealand. 
to describe matroids with an input that is polynomial in the size of the ground set - there are far too many matroids. Because of this, it is common to consider oracles, typically rank oracles. The oracle has a matroid $M$ in mind and we can ask the oracle for the rank of any subset that we care about. A call to the oracle counts as a single step in an algorithm. Seymour [12] showed that, in general, it requires exponentially many calls to a rank oracle to decide if a matroid is binary. Seymour's techniques can be generalised to obtain an analogous negative result for any other field. While this extension is widely known in the matroid community, as far as we know it does not appear in print and we give the details in Section 2 .

The situation for certifying non-representability is much more hopeful. Consider binary matroids. We know [14 that $U_{2,4}$ is the unique excluded minor for binary matroids. It is also the case that a matroid can be proved to have a $U_{2,4}$-minor using eight rank evaluations. Therefore a matroid can be proved to be non-binary using only $O(1)$ rank evaluations. Rota [11] conjectured that for each finite field $\mathbb{F}$, there are, up to isomorphism, only finitely many excluded minors for the class of $\mathbb{F}$-representable matroids. If Rota's Conjecture were true, then it would require only $O(1)$ rank evaluations to certify non-representability for matroids over any finite field. While evidence is accumulating for the truth of Rota's Conjecture, to date it is still only known to hold for $\mathrm{GF}(2), \mathrm{GF}(3)$ [1, 8, 13] and $\mathrm{GF}(4)$ [4]. 1]

An alternative approach to finding a short proof that a matroid is not binary is to attempt to build a binary matrix $A$ with the property that $M$ is binary if and only if $M=M[A]$. Such a matrix can easily be constructed by choosing a basis $B=\left(b_{1}, b_{2}, \ldots, b_{r}\right)$ and considering basic circuits relative to $B$ : the element $a_{i j}$ of $A$ is nonzero if and only if $b_{i}$ is in the basic circuit of the element labelling the $i$ th column of $A$. Moreover, it can be shown that $M \neq M[A]$ with a single rank evaluation. This is an approach that has the potential to be extended to other fields, and does not rely on the truth of Rota's Conjecture.

The problem with extending this technique to other fields is that, in general, a matroid may have many inequivalent representations over a field. Recall that two $\mathbb{F}$-representations of a matroid $M$ are equivalent if one can be obtained from the other by elementary row operations (adding one row to the other, adjoining or deleting a row of zeroes, and scaling a row) and column-scaling. For very small fields one can

\footnotetext{
${ }^{1}$ Since this was written, Geelen, Gerards and Whittle have announced that they have a proof of Rota's Conjecture. However is will be several years before the proof is fully written.
} 
control the number of inequivalent representations of a matroid. Indeed matroids are uniquely representable over $\mathrm{GF}(2)$ and $\mathrm{GF}(3)$. Moreover Kahn [7] showed that 3-connected matroids have at most two inequivalent representations over GF(4) (or are uniquely representable if field automorphisms are allowed). In [10] it is shown that 3-connected matroids have at most six inequivalent representations over GF(5). This is a key ingredient in the proof given in [5] that $O\left(n^{2}\right)$ rank evaluations suffice to prove that a matroid is not $\mathrm{GF}(5)$-representable.

Unfortunately for fields with more than five elements, no bound can be placed on the number of inequivalent representations of 3-connected matroids. But, as noted earlier, it follows from results in [6] that the number of inequivalent representations of a matroid over a fixed prime field $\operatorname{GF}(p)$ becomes bounded if one raises the connectivity somewhat. We also need to ensure that whatever connectivity we are dealing with is possessed by excluded minors for $\operatorname{GF}(p)$. This assurance is a consequence of the previously mentioned result [2] where it is shown that excluded minors over $\operatorname{GF}(p)$ do not have arbitrarily long nested sequences of 3-separations.

\section{Certifying GF $(q)$-Representability}

In this section we give examples showing that there is no succinct certificate for representability over any finite field. Specifically, we show that, for some $\alpha>1$, proving $\operatorname{GF}(q)$-representability for $n$-element matroids requires at least $\alpha^{n}$ rank-values in the worst case. These examples are related to results of Seymour [12] who showed that there is no efficient algorithm to determine whether or not a matroid is binary.

Let $n \geq 3$ be an integer and let $N$ be a rank- $n$ matroid with ground set $\left\{t, a_{1}, b_{1}, a_{2}, b_{2}, \ldots, a_{n}, b_{n}\right\}$ such that

(i) $\left\{t, a_{i}, b_{i}\right\}$ is a triangle for all $i \in\{1,2, \ldots, n\}$, and

(ii) $r\left(\cup_{j \in J}\left\{a_{j}, b_{j}\right\}\right)=|J|+1$ for every proper subset $J$ of $\{1,2, \ldots, n\}$.

Then the matroid $M=N \backslash t$ is a rank-n spike. Each pair $\left\{a_{i}, b_{i}\right\}$ is a leg of the spike. For distinct $i, j \in\{1,2, \ldots, n\}$, the set $\left\{a_{i}, b_{i}, a_{j}, b_{j}\right\}$ is a circuit of $M$. Any other non-spanning circuit of a spike is a transversal of the legs.

Representable spike can be obtained as follows. Let $\mathbb{F}$ be a finite field of order $q \geq 3$ and let $J=\left\{j_{0}, j_{1}, \ldots, j_{n}\right\}$ be a spanning circuit of $\mathrm{PG}(n-1, q)$. Now, for each $i \in\{1,2, \ldots, n\}$, let $a_{i}$ and $b_{i}$ be distinct points of $\mathrm{PG}(n-1, q) \backslash\left\{j_{0}\right\}$ that are on the line spanned by $j_{0}$ and $j_{i}$. Now let $M$ be the restriction of $\mathrm{PG}(n-1, q)$ to $\left\{a_{1}, b_{1}, a_{2}, b_{2}, \ldots, a_{n}, b_{n}\right\}$. 
The next theorem, which is stronger than we need, shows that it really does require a lot of work to pin down a $\mathrm{GF}(q)$-representable spike.

Theorem 2.1. Let $q$ be a prime power and let $M$ be a spike with rank $n>q^{3}$. To prove that $M$ is $\mathrm{GF}(q)$-representable requires at least $2^{\frac{n}{2}}$ rank values.

Let $M$ be a spike with legs $l_{1}=\left\{a_{1}, b_{1}\right\}, \ldots, l_{n}=\left\{a_{n}, b_{n}\right\}$. Let $\mathcal{T}(M)$ denote the set of all dependent transversals of $\left(l_{1}, l_{2}, \ldots, l_{n}\right)$. The following claim is straightforward.

Lemma 2.2. If $T_{1}, T_{2} \in \mathcal{T}(M)$, then $\left|T_{1}-T_{2}\right| \neq 1$.

Proof. If the result fails, then, by symmetry, we may assume that $T_{1}=$ $\left\{a_{1}, \ldots, a_{n}\right\}$ and that $T_{2}=\left\{a_{1}, \ldots, a_{n-1}, b_{n}\right\}$. Thus, $r_{M}\left(T_{1} \cup T_{2}\right)=$ $n-1$. Then, considering the 4 -element circuits $l_{i} \cup l_{n}$, we see that $r(M)=n-1$ contradicting the fact that $r(M)=n$.

The following result is also straightforward; we leave the proof to the reader.

Lemma 2.3. Let $\mathcal{T}$ be a set of transversals of $\left(l_{1}, l_{2}, \ldots, l_{n}\right)$ such that $\left|T_{1}-T_{2}\right| \neq 1$ for any $T_{1}, T_{2} \in \mathcal{T}$. Then there exists a unique spike $M$ on legs $l_{1}, l_{2}, \ldots, l_{n}$ such that $\mathcal{T}(M)=\mathcal{T}$.

The complexity results in this section rely on the following lemma.

Lemma 2.4. Let $M_{1}$ and $M_{2}$ be $G F(q)$-representable spikes with legs $l_{1}, \ldots, l_{n}$ such that $\mathcal{T}\left(M_{2}\right)=\mathcal{T}\left(M_{1}\right) \cup\{T\}$ for some transversal $T \notin$ $\mathcal{T}\left(M_{1}\right)$ of $\left(l_{1}, \ldots, l_{n}\right)$. Then $n \leq(q-1)^{3}+1$.

Proof. We may assume that $T=\left\{a_{1}, a_{2} \ldots, a_{n-1}, b_{n}\right\}$. By Lemma 2.2 , the transversal $\left\{a_{1}, a_{2}, \ldots, a_{n}\right\}$ is independent in $M_{2}$ and, hence, also in $M_{1}$. Consider representations of $M_{1}$ and $M_{2}$; we have:

$$
\begin{aligned}
& A_{1}=\left(\begin{array}{cccccccc}
a_{1} & a_{2} & \cdots & a_{n} & b_{1} & b_{2} & \cdots & b_{n} \\
0 & 0 & \cdots & 0 & 1+\alpha_{1}^{-1} & 1 & \cdots & 1 \\
\vdots & & \ddots & 0 & 1 & 1+\alpha_{2}^{-1} & & 1 \\
0 & 0 & & 1 & 1 & 1 & & 1+\alpha_{n}^{-1}
\end{array}\right) \text { and } \\
& A_{2}=\left(\begin{array}{cccccccc}
a_{1} & a_{2} & \cdots & a_{n} & b_{1} & b_{2} & \cdots & b_{n} \\
1 & 0 & \cdots & 0 & 1+\beta_{1}^{-1} & 1 & \cdots & 1 \\
0 & 1 & & 0 & 1 & 1+\beta_{2}^{-1} & & 1 \\
\vdots & & \ddots & & \vdots & & \ddots & \\
0 & 0 & & 1 & 1 & 1 & & 1+\beta_{n}^{-1},
\end{array}\right)
\end{aligned}
$$


where $\alpha_{i} \neq 0$ and $\beta_{i} \neq 0$ for each $i \in\{1,2, \ldots, n\}$. For $S \subseteq$ $\{1,2, \ldots, n\}$ we let $T_{S}$ denote the transversal $\left\{a_{i}: i \notin S\right\} \cup\left\{b_{i}\right.$ : $i \in S\}$. It is easily verified that $T_{S}$ is dependent in $M_{1}$ if and only if $\sum\left(\alpha_{i}: i \in S\right)=-1$ and that $T_{S}$ is dependent in $M_{2}$ if and only if $\sum\left(\beta_{i}: i \in S\right)=-1$. Suppose, by way of contradiction, that $n>(q-1)^{3}+1$. Note that there are at most $(q-1)^{2}$ distinct pairs $\left(\alpha_{i}, \beta_{i}\right)$. Therefore, there exist $S \subseteq\{1,2, \ldots, n-1\}$, and $\alpha, \beta \in G F(q)-\{0\}$ such that $|S|=q$ and $\left(\alpha_{i}, \beta_{i}\right)=(\alpha, \beta)$ for each $i \in S$. Now, $\sum\left(\alpha_{i}: i \in S \cup\{n\}\right)=q \alpha+\alpha_{n}=\alpha_{n} \neq-1$ and $\sum\left(\beta_{i}: i \in S \cup n\right)=q \beta+\beta_{n}=\beta_{n}=-1$. So $T_{S \cup\{n\}}$ is dependent in $M_{2}$ but not in $M_{1}$, which is a contradiction.

Recall that if $C$ is a circuit-hyperplane of a matroid $M$, then the collection of bases of $M$ together with $C$ is the collection of bases of a matroid $M^{\prime}$, see, for example [9, Proposition 1.5.14]. We say that $M^{\prime}$ is obtained by relaxing $C$.

Proof of Theorem 2.1. Suppose that $M$ has $\operatorname{legs}\left(l_{i}: i=1, \ldots, n\right)$. Let $T$ be a transversal of $\left(l_{1}, l_{2}, \ldots, l_{n}\right)$. If $T \in \mathcal{T}(M)$, then, by Lemma 2.2 and Lemma 2.4, relaxing $T$ results in a non-GF $(q)$-representable spike. Similarly, if $\left|T-T_{1}\right|>1$ for all $T_{1} \in \mathcal{T}(M)$, then restricting $T$ to a circuit results in a non-GF( $(q)$-representable spike. Let $\mathcal{T}^{\prime}$ denote the set of all transversals $T$ of $\left(l_{1}, l_{2}, \ldots, l_{n}\right)$ such that $\left|T-T_{1}\right|>1$ for all $T_{1} \in \mathcal{T}(M)$. Thus, there are $|\mathcal{T}(M)|+\left|\mathcal{T}^{\prime}\right|$ non-GF $(q)$-representable spikes that differ in rank from $M$ only on one set. Moreover, we have $(n+1)\left(|\mathcal{T}(M)|+\left|\mathcal{T}^{\prime}\right|\right) \geq(n+1)|\mathcal{T}(M)|+\left|\mathcal{T}^{\prime}\right| \geq 2^{n}$. Thus $|\mathcal{T}(M)|+$ $\left|\mathcal{T}^{\prime}\right| \geq 2^{n} /(n+1) \geq 2^{\frac{n}{2}}$. Therefore, to distinguish $M$ from each of these non-GF $(q)$-representable matroids, we need at least $2^{\frac{n}{2}}$ rank values.

\section{A SHORT PROOF OF NON-REPRESENTABILITY FOR PRIME FIELDS}

Our short proof of non-representability has three ingredients The first ingredient provides a certificate for a short proof. As noted in the introduction, it is somewhat more general than is required for this paper. We delay its proof until Section 4 .

Theorem 3.1. Let $q$ be a prime power and let $\mathcal{C}$ be a class of matroids such that the following hold.

(i) For all nonempty matroids $M$ in $\mathcal{C}$, there exists $e \in E(M)$ such that either $M \backslash e$ or $M / e$ is in $\mathcal{C}$.

(ii) There exists an integer $t$ such that, if $M \in \mathcal{C}$ and $e \in E(M)$, then the following hold.

(a) If $M / e \notin \mathcal{C}$, then there is a t-separation $(A, B)$ in $M$ such that $e \in \mathrm{cl}_{M}(A-\{e\}) \cap \mathrm{cl}_{M}(B-\{e\})$. 
(b) If $M \backslash e \notin \mathcal{C}$, then there is a t-separation $(A, B)$ in $M$ such that $e \in \mathrm{cl}_{M}^{*}(A-\{e\}) \cap \mathrm{cl}_{M}^{*}(B-\{e\})$.

(iii) There exists an integer $s$ such that each matroid $M \in \mathcal{C}$ has at most $s$ inequivalent representations over $\mathrm{GF}(q)$.

(iv) Each excluded minor for the class of $\mathrm{GF}(q)$-representable matroids belongs to $\mathcal{C}$.

Then proving that an n-element matroid is not representable over $\mathrm{GF}(q)$ requires at most $O\left(n^{2}\right)$ rank evaluations.

We now consider the second ingredient. Let $k \geq 5$ be an integer. Then the definition of what it means for a matroid to be $k$-coherent is given in [6]. In fact $k$-coherence is a connectivity condition intermediate between 3 -connectivity and 4 -connectivity; $k$-coherent matroids are allowed to have 3-separations but only in a controlled way. The full definition takes some preparation and we will not give it here. As a slight weakening of $k$-coherence we say that a matroid is near $k$ coherent if it is connected and either $\operatorname{si}(M)$ or $\operatorname{co}(M)$ is $k$-coherent. The next theorem is [6, Corollary 12.6].

Theorem 3.2. Let $p$ be a prime number and $k \geq 5$ be an integer. Then the following hold.

(i) If $M$ is a nonempty near $k$-coherent matroid, then there is an element $e \in E(M)$ such that either $M \backslash e$ or $M / e$ is near $k$ coherent.

(ii) Let $M$ be a near $k$-coherent and $e \in E(M)$. Then the following hold.

(a) If $M / e$ is not near $k$-coherent, then there is a 4-separation $(A, B)$ in $M$ such that $e \in \mathrm{cl}_{M}(A-\{e\}) \cap \mathrm{cl}_{M}(B-\{e\})$.

(b) If $M \backslash e$ is not near $k$-coherent, then there is a 4-separation $(A, B)$ in $M$ such that $e \in \mathrm{cl}_{M}^{*}(A-\{e\}) \cap \mathrm{cl}_{M}^{*}(B-\{e\})$.

(iii) There is an integer $\mu_{p}$ such that a near $k$-coherent matroid has at most $\mu_{p}$ inequivalent representations over $\mathrm{GF}(p)$.

Our final ingredient is a special case of a theorem of Ben David and Geelen [2]. A nested sequence of 3-separations of length $m$ in a matroid $M$ is a sequence $A_{1}, A_{2}, \ldots, A_{m}=E(M)$ of subsets of $E(M)$ such that, for all $i \in\{1,2, \ldots, m-1\}$, we have $A_{i}$ is a proper subset of $A_{i+1}$ and $\lambda\left(A_{i}\right) \leq 2$.

Theorem 3.3. Let $q$ be a prime power. Then there is an integer $\nu_{q}$ such that an excluded minor for $\mathrm{GF}(q)$-representability has no nested sequence of 3-separations of length $\nu_{q}$.

It is an immediate consequence of the definition of $k$-coherent that a 3-connected matroid with no nested sequence of 3-separations of length 
$k$ is $k$-coherent. From this fact, Theorem 3.3, and the fact that $k$ coherent matroids are near $k$-coherent we obtain the specialisation that we need.

Corollary 3.4. Let $p$ be a prime number. Then an excluded minor for $\mathrm{GF}(p)$-representability is near $\nu_{p}$-coherent.

We now have all the ingredients for the proof of Theorem 1.1. Let $p$ be a prime number and let $\mathcal{C}$ be the class of near $\nu_{p}$-coherent matroids. Consider conditions (i)-(iv) of Theorem 3.1. Condition (i) holds by Theorem 3.2(i). Condition (ii) holds with $t=4$ by Theorem 3.2(ii). Condition (iii) holds with $s=\mu_{p}$ by Theorem 3.2 (iii). Finally condition (iv) holds by Corollary 3.4. Theorem 1.1 now follows from Theorem 3.1.

\section{Proof of Theorem 3.1}

We begin by reviewing basic material on freedom in matroids. The treatment of freedom given here follows [5].

Let $M$ be a matroid. Elements $e$ and $f$ of $M$ are clones if swapping the labels of $e$ and $f$ is an automorphism of $M$. A clonal class of $M$ is a maximal set of elements of $M$ every pair of which are clones. An element $z$ of $M$ is fixed in $M$ if there is no extension of $M$ by an element $z^{\prime}$ in which $z$ and $z^{\prime}$ are independent clones. Dually, an element $z^{\prime}$ of $M$ is cofixed in $M$ if it is fixed in $M^{*}$. Note that if $z$ already has a clone, say $x$, and $\{x, z\}$ is independent, then $z$ is not fixed as we can add a new element freely on the line through $x$ and $z$.

A flat $F$ of $M$ is cyclic if it is a union of circuits of $M$. The next result is straightforward.

Proposition 4.1. Elements $e$ and $f$ of a matroid $M$ are clones if and only if they are contained in the same set of cyclic flats.

Let $e$ and $f$ be elements of $M$. Then $e$ is freer than $f$ if every cyclic flat containing $e$ also contains $f$. Thus $e$ and $f$ are clones if and only if $e$ is freer than $f$ and $f$ is freer than $e$. The freedom of an element $e$ of $M$ is the maximum size of an independent clonal class containing $e$ among all extensions of $M$. This maximum does not exist if and only if $e$ is a coloop of $M$; in this case the freedom of $e$ is infinity. Observe that an element is fixed in $M$ if and only if its freedom is 0 or 1 .

The notion of freedom of an element in a matroid was introduced by Duke [3]. While his definition is different from that given here, it is, in fact, equivalent; see [5, Lemma 2.8]. The next lemma is [3, Theorem 6.2]. A proof is also given in [5, Lemma 2.9]. 
Lemma 4.2. Let $a$ and $b$ be elements of the matroids $M$ such that $a$ is freer than $b$. Then the freedom of $a$ is at least the freedom of $b$. Moreover, either $a$ and $b$ are clones or the freedom of $a$ is greater than the freedom of $b$.

For elements $e$ and $f$ of a matroid $M$, it is easily seen that the freedom of $f$ does not decrease when we delete $e$. Contraction has a slightly more complicated effect on freedom. The proof of part (ii) of the next lemma is given in [5, Lemma 2.10].

Lemma 4.3. Let $e$ and $f$ be elements of the matroid $M$ where $f$ has freedom $k$ in $M$.

(i) The freedom of $f$ in $M \backslash e$ is at least $k$.

(ii) The freedom of $f$ in $M / e$ is at least $k-1$. Moreover, if $f$ has freedom $k-1$ in $M / e$, then $f$ is freer than e in $M$.

The cofreedom of an element $e$ of $M$ is the freedom of $e$ in $M^{*}$. It is easily seen that the cyclic flats of $M^{*}$ are the complements of the cyclic flats of $M$. It follows from this fact that $e$ is freer than $f$ in $M^{*}$ if and only if $f$ is freer than $e$ in $M$. Note that an element is cofixed in $M$ if and only if its cofreedom is either 0 or 1 . The next lemma is the dual of Lemma 4.3 .

Lemma 4.4. Let $e$ and $f$ be elements of the matroid $M$ where $f$ has cofreedom $k$ in $M$.

(i) The cofreedom of $f$ in $M / e$ is at least $k$.

(ii) The cofreedom of $f$ in $M \backslash e$ is at least $k-1$. Moreover, if $f$ has cofreedom $k-1$ in $M \backslash e$, then $e$ is freer than $f$ in $M$.

We omit the easy proof of the next observation.

Lemma 4.5. The ground set of a matroid $M$ consists of a single clonal class if and only if $M$ is uniform. Moreover, if $M$ is uniform and $r(M), r^{*}(M)>0$, then each element of $M$ has freedom $r(M)$ and cofreedom $r\left(M^{*}\right)$.

Elements $a$ and $b$ of a matroid $M$ are incomparable if $a$ is not freer than $b$ and $b$ is not freer than $a$. Note that an element $a$ has freedom 0 if and only if it is a loop of $M$ and has cofreedom 0 if and only if it is a coloop of $M$.

Lemma 4.6. Let $a$ be an element of the matroid $M$ that is neither a loop nor a coloop. If the freedom of a is $\gamma$ and the cofreedom of a is $\delta$, then $M$ has a $U_{\gamma, \gamma+\delta}$-minor. 
Proof. Note that $\gamma, \delta>0$. Assume that $M$ is not uniform. By Lemma 4.5, $M$ has an element $b$ such that $a$ and $b$ are not clones. Then either (i) $a$ and $b$ are incomparable, (ii) $a$ is strictly freer than $b$, or (iii) $b$ is strictly freer than $a$.

Assume that either (i) or (ii) holds and consider the matroid $M \backslash b$. Then, by Lemma 4.3(i), $a$ has freedom at least $\gamma$ in $M \backslash b$ and by Lemma 4.4(ii), $a$ has cofreedom at least $\delta$ in $M \backslash b$. As $\gamma, \delta>0$, we see that $a$ is neither a loop nor a coloop of $M \backslash b$. On the other hand, if (iii) holds, then we apply Lemma 4.3(ii) and Lemma 4.4(i) to obtain the same conclusion for $M / b$.

Iterating the above procedure we eventually arrive at a minor $N$ of $M$ using $a$ whose ground set consists of a single clonal class, where the freedom of $a$ in $N$ is $\gamma^{\prime} \geq \gamma$ and the cofreedom is $\delta^{\prime} \geq \delta$. By Lemma 4.5 $N$ is uniform, indeed $N \cong U_{\gamma^{\prime}, \delta^{\prime}+\gamma^{\prime}}$. The lemma now follows from the fact that $U_{\gamma^{\prime}, \delta^{\prime}+\gamma^{\prime}}$ has a $U_{\gamma, \gamma+\delta^{\prime}}$-minor.

Corollary 4.7. Let $q$ be a prime power and let $M$ be a matroid that is either $\mathrm{GF}(q)$-representable or is an excluded minor for $\mathrm{GF}(q)$ representability.

(i) If e is not fixed in $M$, then e has cofreedom at most $q$.

(ii) If $e$ is not cofixed in $M$, then e has freedom at most $q$.

Proof. Assume that $e$ is not fixed in $M$. If $e$ is a coloop, then $e$ has cofreedom 0, so that (i) holds. Assume that $e$ is not a coloop of $M$. Then $e$ has freedom $k \geq 2$. Assume that $e$ has cofreedom $q^{\prime}>q$. By Lemma 4.6, $M$ has a $U_{k, q^{\prime}+k^{-}}$minor and hence a $U_{2, q+3}$-minor. But this matroid is neither $\operatorname{GF}(q)$-representable nor an excluded minor for $\operatorname{GF}(q)$ contradicting the choice of $M$. Thus (i) holds in this case too. Part (ii) is the dual of (i).

Recall that the connectivity function $\lambda_{M}$ of a matroid $M$ on $E$ is defined, for all subsets $X$ of $E$ by $\lambda_{M}(X)=r(X)+r(E-X)-r(M)$. We say that a partition $(X, E-X)$ of $E$ is a $k$-separation if $\lambda_{M}(X)<k$. If $\lambda_{M}(X)=k-1$, then the $k$-separation is exact. Recall also that the coclosure operator of $M$, denoted $\mathrm{cl}^{*}$ is the closure operator of $M^{*}$. Thus $x \in \mathrm{cl}^{*}(A)$ if and only if $x \in \mathrm{cl}_{M^{*}}(A)$.

Lemma 4.8. Let $e$ be an element of the matroid $M$ and $(A, B)$ be a $(t+1)$-separation.

(i) If $e \in \operatorname{cl}(A-\{e\}) \cap \operatorname{cl}(B-\{e\})$, then e has freedom at most $t$ in $M$.

(ii) If $e \in \operatorname{cl}^{*}(A-\{e\}) \cap \mathrm{cl}^{*}(B-\{e\})$, then e has cofreedom at most $t$ in $M$. 
Proof. Consider (i). Let $M^{\prime}$ be an extension of $M$ by a set $Z$ such that the members of $Z \cup\{e\}$ are clones. Then $Z \cup\{e\}$ is contained in $\mathrm{cl}_{M^{\prime}}(A) \cap \mathrm{cl}_{M^{\prime}}(B)$. It now follows from the submodularity of the rank function that $r_{M^{\prime}}(Z \cup\{e\}) \leq t$ so that $e$ has freedom at most $t$ in $M$. Part (ii) is the dual of (i).

The next lemma is [5, Theorem 4.1]. As the result is vital we repeat the short proof here. It shows that, if an element $e$ has bounded freedom, then the candidates for a possible extension of a representation of $M \backslash e$ to a representation of $M$ all belong to a bounded rank subspace of the projective geometry. If none of these candidates succeed, then either $M$ is not representable, or a representation of $M$ can only be obtained by extending some other representation of $M \backslash e$.

Lemma 4.9. Let e be an element of a rank-r matroid $M$. Let $R$ be a $\mathrm{GF}(q)$-representation of $M \backslash$ e considered as a restriction of $\mathrm{PG}(r-1, q)$. Let $K$ be a flat of $\mathrm{PG}(r-1, q)$ such that, for each flat $F$ of $M$ in which $e$ is not a coloop, $e \in F$ if and only if the flat of $\mathrm{PG}(r-1, q)$ that is spanned by $F-\{e\}$ contains $K$. Then the rank of $K$ is at most the freedom of $e$ in $M$.

Proof. Let $\mathbb{F}$ be an infinite extension field of $\mathrm{GF}(q)$ and let $\mathcal{P}$ be the projective space of rank $r$ over $\mathbb{F}$. Thus $\mathcal{P}$ contains $\mathrm{PG}(r-1, q)$. Let $K^{\prime}$ be the flat of $\mathcal{P}$ that is spanned by $K$. Then, for each flat $F$ of $M$ in which $e$ is not a coloop, $e$ is in $F$ if and only if the flat of $\mathcal{P}$ that is spanned by $F-\{e\}$ contains $K^{\prime}$. Let $K^{*}$ denote the set of points $x$ of $K^{\prime}$ for which $R \cup\{x\}$ is an $\mathbb{F}$-representation of $M$. Note that an element $x$ of $K^{\prime}$ is in $K^{*}$ if and only if, for each flat $F$ of $M$ not containing $e$, the point $x$ is not contained in the flat of $\mathcal{P}$ spanned by $F$. There is a finite number of flats of $M$ that do not contain $e$. Therefore, by a simple comparison of measures, $K^{*}$ spans $K^{\prime}$. It is now straightforward to deduce that $K^{*}$ is spanned by some independent set $S$ such that $S$ is a clonal class of the matroid $M^{\prime}$ that is represented by $R \cup S$. Note that $M^{\prime}$ is an extension of $M$ so that $|S|$ is at most the freedom of $e$ in $M$.

Proof of Theorem 3.1. Let $M$ be a matroid that is not representable over $\operatorname{GF}(q)$. In what follows, suppose that we have a Claimant whose brief is to succinctly prove to an Adjudicator that $M$ is not GF $(q)$-representable. The Claimant knows everything about $M$ but can only reveal quadratically many rank-values to the Adjudicator. The Claimant can find a minimal minor $M^{\prime}=M \backslash D / C$ of $M$ that is not $\mathrm{GF}(q)$-representable. Now, for any $X \subseteq E\left(M^{\prime}\right)$, we have 
$r_{M^{\prime}}(X)=r_{M}(X \cup C)-r_{M}(C)$; thus one rank evaluation for $M^{\prime}$ requires only two rank evaluations for $M$ (and if we need to make multiple rank evaluations for $M^{\prime}$, we only need to compute $r_{M}(C)$ once). The Adjudicator concedes that it suffices to show that $M^{\prime}$ is not $\operatorname{GF}(q)$ representable. Thus we lose no generality in assuming that $M$ is an excluded minor for $\mathrm{GF}(q)$-representability.

Let $l=\max \{t, q\}$.

4.9.1. If $N$ is a nonempty member of $\mathcal{C}$ and $N$ is either an excluded minor for $\mathrm{GF}(q)$-representability or is $\mathrm{GF}(q)$-representable, then there is an element $e$ of $N$ such that either:

(a) $N \backslash e \in \mathcal{C}$ and e has freedom at most $l$, or

(b) $N / e \in \mathcal{C}$ and e has cofreedom at most $l$.

Proof. By property (i) of $\mathcal{C}$, there is an element $e \in E(N)$ such that either $N \backslash e$ or $N / e$ is in $\mathcal{C}$. Up to duality we may assume that $N \backslash e$ is in $\mathcal{C}$. If the freedom of $e$ in $N$ is at most $l$, then the claim holds. Assume otherwise. By Corollary 4.7, $e$ is cofixed in $N$. Assume that $N / e \notin \mathcal{C}$. Then, by property (ii) of $\mathcal{C}$, there is a $t$-separation $(A, B)$ in $N$ such that $e \in \operatorname{cl}(A-\{e\}) \cap \operatorname{cl}(B-\{e\})$. Then, by Lemma 4.8, $e$ has freedom at most $t \leq l$. This contradiction shows that $N / e \in \mathcal{C}$. As $e$ is cofixed it has cofreedom $1<l$. Thus (b) is satisfied by $e$.

By property (iv) of $\mathcal{C}$ our excluded minor $M$ belongs to $\mathcal{C}$. Say $|E(M)|=k$. By 4.9.1 the Claimant can find a sequence $M_{0}, M_{1}, \ldots, M_{k}=M$ of matroids in $\mathcal{C}$ such that $M_{0}$ is empty, and for each $i \in\{1,2, \ldots, k\}$ there is an element $e_{i} \in M_{i}$ such that either

$$
\begin{aligned}
& M_{i-1}=M_{i} \backslash e_{i} \text { and } e_{i} \text { has freedom at most } l \text { in } M_{i}, \text { or } \\
& M_{i-1}=M_{i} / e_{i} \text { and } e_{i} \text { has cofreedom at most } l \text { in } M_{i} .
\end{aligned}
$$

For each $i \in\{1,2, \ldots, k\}$, let $\mathcal{R}_{i}$ be a complete set of inequivalent $\mathrm{GF}(q)$-representations of $M_{i}$; that is, any $\mathrm{GF}(q)$-representation of $M_{i}$ is equivalent to some representation in $\mathcal{R}_{i}$, but no two representations in $\mathcal{R}_{i}$ are equivalent. By property (iii) of $\mathcal{C}$, we have $\left|\mathcal{R}_{i}\right| \leq s$ for all $i \in\{1,2, \ldots, k\}$. Moreover, since $M$ is not $\mathrm{GF}(q)$-representable, $\mathcal{R}_{k}$ is empty. The Claimant, who knows everything about $M$, can determine $\left(\mathcal{R}_{1}, \mathcal{R}_{2}, \ldots, \mathcal{R}_{k}\right)$. The Claimants proof will consist of the sets $\left(\mathcal{R}_{1}, \mathcal{R}_{2}, \ldots, \mathcal{R}_{k}\right)$ along with a recursive argument that each representation of $M_{i}$ is equivalent to one in $\mathcal{R}_{i}$. Note that we do not have to prove that members of $\mathcal{R}_{i}$ are, in fact, representations of $M_{i}$, only that these are the only possible candidates for representations of $M_{i}$.

Suppose that the Adjudicator is already satisfied that each $\operatorname{GF}(q)$ representation of $M_{i-1}$ is equivalent to some representation in $\mathcal{R}_{i-1}$. By duality we may assume that $M_{i-1}=M_{i} \backslash e_{i}$. Consider a representation 
$R_{i}$ of $M_{i}$. Evidently this representation is obtained by extending a representation $R$ of $M_{i-1}$. For each such representation we need to identify all possible points $P$ in $\mathrm{PG}(r-1, q)$ such that $R \cup\{p\}$ represents $M_{i}$ for $p \in P$. Suppose that $S \subseteq E\left(M_{i}\right)-\left\{e_{i}\right\}$ with $e_{i} \in \mathrm{cl}_{M_{i}}(S)$. Then the set $P$ is in the subspace of $\mathrm{PG}(r-1, q)$ spanned by $S$. The Claimant will try to identify $P$ by considering the intersection of all such flats. This is done inductively. The Claimant constructs a sequence $K_{0}, K_{1}, \ldots, K_{m}$ of subspaces of $\mathrm{PG}(r-1, q)$ as follows. Let $K_{0}=P G(r-1, q)$. For the flat $K_{j}$ one of the following holds.

1. There is a set $S_{j} \subseteq E\left(M_{i}\right)-\{e\}$ and an element $a_{j}$ of $K_{j}$ such that $e_{i}$ is in the closure of $S_{j}$ in $M_{i}$ and $a_{j}$ is not spanned by $S_{j}$ in $\mathrm{PG}(r-1, q)$. In this case, the Claimant defines $K_{j+1}$ to be the intersection of $K_{j}$ with the flat of $\mathrm{PG}(r-1, q)$ spanned by $S_{j}$.

2. For each flat $F$ of $M_{i}$ containing $e_{i}$ such that $e_{i}$ is not a coloop of $M_{i} \mid F$, the flat $K_{j}$ is contained in the flat of $\mathrm{PG}(r-1, q)$ that is spanned by $F-\{e\}$. Then $j=m$.

Note that $m \leq r$ and $K_{m}$ contains the set $P$ (which may be empty). The Claimant reveals the sets $\left(S_{0}, S_{1}, \ldots, S_{m-1}\right)$ to the Adjudicator. Then, by revealing $O(r)$ rank values the Claimant convinces the Adjudicator that $e_{i}$ is in the closure of each of $S_{0}, S_{1}, \ldots, S_{m-1}$. Given $S_{0}, S_{1}, \ldots, S_{m-1}$, the Adjudicator can then determine $K_{0}, K_{1}, \ldots, K_{m}$ efficiently using routine linear algebra. Now we are in one of the following cases.

Case 1. There is a set $S \subseteq E\left(M_{i}\right)-\left\{e_{i}\right\}$ such that $e_{i}$ is not in the closure of $S$ in $M_{i}$ but $K_{m}$ is contained in the flat spanned by $S$ in $\mathrm{PG}(r-1, q)$.

Case 2. For each flat $F$ of $M_{i}$ that does not contain $e_{i}$, the flat $K_{m}$ is not contained in the flat of $\operatorname{PG}(r-1, q)$ that is spanned by $F$.

In Case 1 the Claimant can easily convince the Adjudicator $R$ cannot be extended to a representation of $M_{i}$. Indeed, two rank-values satisfy the Adjudicator that $e \in \mathrm{cl}_{M_{i}}(S)$ and the Adjudicator can check that $K_{m}$ is spanned by $S$. Now consider the second case. As $e_{i}$ has cofreedom at most $l$, it follows from Lemma 4.9 that $K_{m}$ has rank at most $l$. If $K_{m}$ has rank 0, then the Adjudicator concedes that $R$ cannot be extended to a representation of $M_{k}$. Suppose that $K_{m}$ is nonempty. Then $K_{m}$ has at most $\frac{q^{l}-1}{q-1}$ elements. For an element of $K_{m}$ that does not extend $R$ to a representation of $M_{i}$, the Claimant can reveal a single rank value to expose the fault, that is, the elements of $K_{m}$ that are not in $P$ can be identified with $O(1)$ rank evaluations. 
As $\mathcal{R}_{i-1}$ has at most $s$ members, we need only $O(r)$ rank evaluations to determine $\mathcal{R}_{i}$ from $\mathcal{R}_{i-1}$. Therefore $O\left(|E(M)|^{2}\right.$ ) rank evaluations suffice to prove that $M$ is not $\operatorname{GF}(q)$-representable.

\section{REFERENCES}

[1] R. E. Bixby, On Reid's characterisation of the ternary matroids, J. Combin. Theory Ser. B, 26 (1979) 174-204.

[2] S. Ben-David and J. Geelen, On Rota's Conjecture and nested separations in matroids, to appear in J. Combin. Theory Ser. B.

[3] R. Duke, Freedom in matroids, Ars Combin. 26B (1988) 191-216.

[4] J. Geelen, B. Gerards, and A. Kapoor, The excluded minors for GF(4)representable matroids, J. Combin. Theory Ser. B, 79 (2000) 247-299.

[5] J. Geelen, J. G. Oxley, D. Vertigan, and G. Whittle, A short proof of nonGF(5)-representability of matroids, J. Combin. Theory Ser. B, 91 (2004) 105121.

[6] J. Geelen and G. Whittle, Inequivalent representations of matroids over prime fields, Advances in Applied Math. 51 (2013), 1-175.

[7] J. Kahn, On the uniqueness of matroid representations over GF(4), Bull. London Math. Soc. 20 (1988), 5-10.

[8] J. Kahn and P. D. Seymour, On forbidden minors for GF(3), Proc. Amer. Math. Soc., 102 (1988) 437-440.

[9] J. Oxley, Matroid Theory, Oxford University Press, New York, 2011.

[10] J. Oxley, D. Vertigan, and G. Whittle, On inequivalent representations of matroids over finite fields, J. Combin. Theory Ser. B, 67 (1996) 325-343.

[11] G. -C. Rota, Combinatorial theory, old and new, in: Proceedings of the International Congress on Mathematics, Nice, September 1970, Gauthier, Chichester, 1970, pp. 229-233.

[12] P. D. Seymour, Recognising graphic matroids, Combinatorica 1 (1981) 75-78.

[13] P. D. Seymour, Matroid representation over GF(3), J. Combin. Theory Ser. $B, 26$ (1979) 159-173.

[14] W. T. Tutte, A homotopy theorem for matroids, I, II, Trans. Amer. Math. Soc. 88 (1958) 144-177.

Department of Combinatorics and Optimization, University of Waterloo, Waterloo, Ontario, Canada

E-mail address: jfgeelen@math. uwaterloo.ca

School of Mathematics and Statistics, Victoria University of Wellington, New Zealand

E-mail address: geoff.whittle@vuw.ac.nz 